\title{
The balance of power: innate lymphoid cells in tissue inflammation and repair
}

\author{
Jim G. Castellanos and Randy S. Longman \\ jill Roberts Institute for Research in IBD, Division of Gastroenterology and Hepatology, Department of Medicine, Weill Cornell Medicine, New York, New York, USA.
}

\begin{abstract}
Over the last ten years, immunologists have recognized the central importance of an emerging group of innate lymphoid cells (ILCs) in health and disease. Characterization of these cells has provided a molecular definition of ILCs and their tissuespecific functions. Although the lineage-defining transcription factors, cytokine production, and nomenclature parallel those of T helper cells, ILCs do not require adaptive immune programming. Both environmental and host-derived signals shape the function of these evolutionarily ancient cells, which provide pathogen protection and promote tissue restoration. As such, ILCs function as a double-edged sword, balancing the inflammatory and reparative responses that arise during injury and disease. This Review highlights our recent understanding of tissue-resident ILCs and the signals that regulate their contribution to inflammation and tissue repair in health and disease.
\end{abstract}

\section{Introduction}

Recent advances in cellular and molecular immunology have revealed the existence of a broad class of innate lymphoid cells (ILCs). ILCs are evolutionarily ancient cells, present in common ancestors of both jawless and jawed vertebrates, which endow the primordial immune system with the capacity for rapid defense against pathogens $(1,2)$. An array of ILC effectors have emerged to balance the collateral damage from sustained inflammation and to promote tissue restoration for overall organismal protection. Similarly to conventional T helper cells, ILCs can be classified by their lineage-defining transcription factors and effector cytokines; however, in contrast to T helper cells, ILCs do not require conventional adaptive programming. Instead, as primarily tissue-resident cells, environmental and organ-specific cues shape their effector functions and spatial location, enabling rapid modulation of host pathophysiology. This Review highlights the regulatory factors that drive tissue homeostasis of ILCs as they balance pathogen defense, tissue repair, and chronic inflammation. A better understanding of this complex biology will help address the diagnostic and therapeutic potential of ILCs in health and disease.

\section{ILC development and subset function}

All ILC development requires signaling through the common $\gamma$ chain of the IL-2 receptor as well as inhibitor of DNA 2-dependent (ID2-dependent) differentiation from a common lymphoid progenitor $(3,4)$. Functionally, ILCs can be divided into cytolytic and noncytolytic ILCs. Cytolytic ILCs, also referred to as conventional NK (cNK) cells, release cytolytic effector molecules including perforin and granzyme B, which can kill tumor or virus-infected

Conflict of interest: RSL serves on the scientific advisory board of Ancillia Inc. and as a consultant for Takeda. RSL also receives research grant support from Boehringer Ingelheim.

Copyright: (c) 2019, American Society for Clinical Investigation.

Reference information: / Clin Invest. 2019;129(7):2640-2650.

https://doi.org/10.1172/JCI124617. tissue. In contrast to cNKs, noncytolytic or "helper" ILCs arise from a GATA-3-dependent common helper innate lymphoid precursor (CHILP) $(5,6)$. Helper ILCs are generally classified into subgroups according to their cytokine and transcription factor expression, which parallels $\mathrm{T}$ helper cell subsets: group 1 (ILC1), group 2 (ILC2), and group 3 (ILC3) $(7,8)$.

ILC1s. ILC1s are a phenotypically heterogeneous group of tissue-resident cells located in the intestine, liver, uterus, and salivary gland (9-11). These cells are characterized by the production of type 1 cytokines, including IFN- $\gamma$, and require T-BET expression. In contrast to cytotoxic cNKs, ILC1s are tissueresident cells that do not require the T-box transcription factor eomesodermin (EOMES) for development and lack the MHC I-specific inhibitory receptors that guide cNK cytolytic function (11). Additional tissue- and organ-specific features of ILC1s also exist; for example, intraepithelial ILC1s reside in mucosal tissue and develop independently of IL-15, but require both EOMES and T-BET (12). Moreover, tissue-specific cues, including TGF- $\beta$, may regulate plasticity between $\mathrm{cNKs}$ and $\mathrm{TNF}-\alpha$-producing ILC1s, illustrating the diversity and heterogeneity of ILC1s $(13,14)$.

ILC2s. ILC2s are systemically dispersed in lymphoid and nonlymphoid tissues, including the brain, heart, lung, kidney, skin, intestine, and adipose tissue, where they play a central role in protection from parasitic infection, allergic inflammation, and local tissue repair (15-17). ILCs are characterized by the production of the type 2 cytokines IL- 5 and IL-13, and the transcription factor GATA-3 is critical for ILC2 development in both mice and humans $(5,18)$. ILC2s express receptors that respond to secreted factors in the epithelium, including IL-25, IL-33, TSLP, and prostaglandin $\mathrm{D}_{2}$ (CRTh2). ILC2s play a key role in controlling both eosinophil homeostasis and allergic response through constitutive and inducible production of IL-13 in the intestine and lung, respectively (16). In adipose tissue, IL-25 and IL-33 trigger infiltration of ILC2s and subsequent regulation of IL-13-dependent inflammation (19), as well as "beiging" of adipose tissue (20) to increase energy consumption and limit obesity. 
ILC3s. ILC3s are most abundant at mucosal barrier surfaces. They are characterized by their expression and dependence on the transcription factor $\operatorname{ROR} \gamma t(7,21)$. Lymphoid tissue inducer (LTi) cells, the prototypical ILC3 subtype, are critical for lymph node and Peyer's patch organogenesis (22). In addition to mucosal lymphoid structure development, LTi cells reorganize lymphoid tissue following infection (23) and promote adaptive barrier immunity in adult organisms $(24,25)$. Although LTi cells were discovered decades ago, more recent studies have revealed the presence of mucosal tissue ILCs that produce the Th17-related cytokines IL-22 and IL-17 in response to IL-1 $\beta$ and IL-23 stimulation $(26,27)$. The commensal microbiota plays a key role in shaping the function of these cells during homeostasis and during intestinal inflammation $(28,29)$. These tissue-resident ILC3s can be further subdivided into CCR6 ${ }^{+}$LTi-like ILC3s and NCR ${ }^{+}$T-BET ${ }^{+}$ILC3s $(30,31)$.

Plasticity between ILC subsets provides another level of immune regulation and can be shaped by tissue-dependent cues (32). Fate-mapping studies have revealed that $\mathrm{T}^{-B E T}{ }^{+}$ILC3s maintain functional plasticity with ILC1s, as T-BET expression downregulates ROR $\gamma \mathrm{t}$, leading to an inflammatory phenotype associated with IFN- $\gamma$ production $(30,33)$. Evidence of transdifferentiation from ILC3 to ILC1 as well as ILC2 to ILC1 has also been reported in Crohn's disease and chronic obstructive pulmonary disease $(10,34,35)$. In addition, a potentially separate subset of regulatory ILCs producing IL-10 have been reported in both mouse and human $(36,37)$. Many of these ILC subsets and precursors also exist in circulating blood and secondary lymphoid tissue of humans $(38,39)$. Given the role for sustained cytokine exposure in driving this plasticity in vitro (40), it is likely that tissuespecific factors in both autoimmune and infectious disease shape ILC function locally.

\section{Tissue-specific regulation of ILCs}

Tissue-specific cues shape the function of adult ILCs in situ. ILC precursors seed the fetal intestine and possess the capacity to develop into all ILC lineages $(41,42)$. Genetic and parabiosis experiments have revealed evidence for the local proliferation of tissue-resident ILCs $(43,44)$. In particular, factors in the local microenvironment (including interferon and IL-27) and availability of IL-2 shape the development and function of ILC2s during helminth challenge. This tissue maturation of ILCs imprints subsets with distinct patterns of activating receptors, which enable them to respond to tissue-specific perturbations, including injury and tumor $(45,46)$. While these studies provide support for a tissue-resident ILC, the migratory capacity of ILCs remains a matter of debate (47), as the imprinted tissue homing of ILCs has been reported in both the skin and the gut $(48,49)$. Mechanistically, retinoic acid (RA) drives $\alpha_{4} \beta_{7}$ - and CCR9-dependent homing of ILC1s and ILC3s, whereas ILC2s acquire these receptors in the bone marrow (49). Tissue-resident macrophages in the gut express CXCL16, which actively retains and enables spatial colocalization with CXCR6 ${ }^{+} \mathrm{NKp}_{4} 6^{+}$ILC3s in the mucosal tissue (50). Transcriptional analysis of tissue-resident lymphocytes revealed a role for the transcription factors HOBIT and BLIMP1 in enforcing tissue residence by repressing Klf2, S1pr1, and Ccr7 (51), but the molecular mechanisms accounting for tissue residency in ILC subsets remain unknown. Recent studies have revealed emerging roles for the microbial and neural-derived factors in tissue-specific regulation of ILCs.

Microbial and metabolic regulation of tissue ILC function. The commensal microbiota and their metabolites play an important role in regulating ILC numbers and function. Although LTi cells develop in the absence of microbiota, the reduced number (29) and production of IL-22 (28) by ILC3s in germ-free mice lead to an increased susceptibility to inflammation. Mucosal association and/or lymphoid tissue residence are particular features of the microbial taxa that shape intestinal ILC3 responses (52-55). ILC2s can also develop in the absence of microbiota (56). Given the overall reduction in lymphocytic cellularity in germ-free mice, the higher proportion of ILC2s can skew the overall mucosal immune response (57). In addition to changes in cellularity, microbiota can induce transcriptional and epigenetic modifications underlying plasticity. High-resolution analysis of murine ILC subsets revealed that the removal of microbial signals diminished lineage-specific methylation marks (58). Following antibiotic treatment, both mucosal ILC1s and ILC2s gain transcriptional elements associated with ILC3s. Moreover, Il17a was reduced across all subsets and particularly in Tbx21-expressing clusters of ILC3s. This emerging molecular characterization is consistent with functional reports showing the potential role for the microbiome in ILC plasticity $(30,59)$ and may provide insight into therapeutic targets for regulating ILC function.

Specific microbial and dietary metabolites have also emerged as critical regulators of ILC development and function. All intestinal ILCs express the aryl hydrocarbon receptor (Ahr), which binds tryptophan-derived ligands from either dietary, bacterial, or endogenous sources $(60,61)$. Ahr ligands are critical for the postnatal expansion of intestinal ILC3s and the subsequent formation of intestinal lymphoid follicles $(60,62,63)$. Ahr ligands enable ILC3 regulation of the commensal microbiota and homeostatic immunity (64). In contrast, cell-intrinsic Ahr inhibits key ILC2 effectors (65). This Ahr dependency establishes a link between dietary nutrients and the balance between ILC2 and ILC3 immunity in the gut. Host catabolism of dietary tryptophan can tune this response by modulating the availability of these Ahr ligands and enhancing IL-22-dependent colonization resistance (66). The timing of Ahr ligand stimulation can be critical. Transient colonization of pregnant dams is sufficient to induce macrophage and ILC3 recruitment to the intestine of progeny in utero, which subsequently limits inflammation induced by infectious challenge (67). Signaling from dietary-derived Ahr ligands is, therefore, a critical factor limiting inflammation.

In a similar fashion, dietary retinoids regulate both transcription and development of tissue ILCs. RA binding to intracellular receptors induces receptor-ligand complexes that transfer to the nucleus and bind to RA response elements (68). These response elements directly regulate both Rorc and Il22 in type 3 immune responses. The availability of retinoids in utero also plays a key role in regulating population size of LTi and ILC3s. The progeny of mice that lack exposure to retinoids during this crucial period show increased susceptibility to viral infection (69).

In adult mice, nutritional status also regulates ILC-mediated immunity. Overall caloric intake can directly regulate ILC2 function through the release of vasoactive intestinal peptide (VIP) (16). 
VIP signaling through VIP receptor type 2 on ILC2s can induce IL-5and IL-13-dependent eosinophil recruitment. The link between caloric intake and basal eosinophil response to allergic challenge opens a window for helminthic parasites to exploit during periods of reduced caloric intake or malnutrition. The co-occurrence of vitamin A deficiency with chronic malnutrition activates a mechanism that attempts to limit this ILC2 defect. Specifically, vitamin A deficiency promotes the accumulation of mucosal ILC2s and increased production of IL-13 (70). This response allows the host to sustain barrier immunity to helminth infection despite malnutrition. Expression of the RA receptor RXR $\gamma$ and PPAR $\gamma$ by ILC2s may mediate the direct sensing of vitamin A metabolites (61). ILC2 expression of additional genes involved in lipid sensing and fatty acid metabolism may similarly help to reduce barrier inflammation when dietary nutrients are limited (71).

Collectively, these pathways provide the circuitry linking nutrient availability to immune status, and this link is further imprinted by metabolic inflammatory checkpoints in ILCs (72). Next-generation sequencing platforms have revealed more extensive mechanisms for ILC subset metabolic regulation during inflammation, including mTOR and Notch signaling in ILC1s, sphingolipid and amino acid metabolism in ILC2s, and carbohydrate metabolism in ILC3s (58). Further studies are needed to functionally validate these findings.

Neuronal control of ILC immunity. The nervous system also provides critical signaling for guiding ILC functions in tissue. Neural-derived ligands for RET direct the fetal induction of lymphoid tissue organization in the intestine (73), and neuronal release of VIP links caloric intake with ILC2 immunity. These findings reflect close interaction of neural and immune pathways, which enable neuronal regulation of mucosal ILC immunity. ILC2s colocalize with cholinergic neurons in the lung and gut tissue (74-76). ILC2-specific expression of the receptor for neuromedin U (NMU) promotes ILC2 proliferation and type 2 cytokine production in synergy with IL-25. This cholinergic signal is required for eosinophil recruitment and helminth expulsion in mouse models. In addition, ILC2s colocalize with pulmonary neuroendocrine cells (PNECs) near airway branch points (77). PNEC production of calcitonin gene-related peptide (CGRP) triggers ILC2s and amplifies allergic asthma responses. This stimulatory input is balanced by adrenergic input through the $\beta_{2}$-adrenergic receptor ( $\beta 2 \mathrm{AR})$, which is also highly expressed on ILC2s in intestinal tissue (78). $\beta 2 A R$ signals repress ILC2 proliferation, cytokine production, and response to helminth infection. Together, these findings illustrate a critical role for neuronal signals in balancing ILC2 responses in the tissue.

In addition, neuronal signals converge on tissue ILC3s to limit inflammation. Enteric glial cells maintain spatial colocalization with ILC3s in the adult mouse intestine. Glial cells release GDNF family ligands in response to MYD88-dependent microbial signals, which subsequently trigger ILC3-autonomous RET-dependent production of IL-22 (79). This glial-ILC3 circuit limits inflammation and promotes IL-22-dependent tissue repair in mouse models of chemical and infectious colitis. Neural signals also promote ILC3-dependent tissue resolution independent of IL-22. Vagal nerve-derived acetylcholine upregulates production of the protective immunoresolvent molecule PCTR1 by ILC3s (80). The same study revealed that delayed tissue resolution following E. coli infection could be restored following either PCTR1 or ILC3 administration. Collectively, these findings reveal neuroimmune regulation of ILC3 function in balancing tissue inflammation and restoration. The functional role for these pathways in ILCs and their ability to serve as pharmacologic targets in humans need to be assessed.

\section{Tissue ILCs limit infection-triggered mucosal inflammation}

The close proximity of tissue ILCs to barrier surfaces - in the skin, gut, and lung - not only facilitates rapid responses against invading pathogens but also limits rampant inflammation (Figure 1). Distinct mechanisms and functions are associated with each group of ILCs at the mucosal barrier.

As primary producers of mucosal IL-22, intestinal ILC3s play a critical role in protecting against gut bacterial infections $(28,81$, 82). In response to IL-22, epithelial cells secrete antimicrobial peptides (REG3G, REG3B), lipocalin, and mucus to promote barrier protection against microbial damage. IL-22 can be triggered in the steady state by epithelial-adherent commensal microbiota, including segmented filamentous bacteria and adherent-invasive E. coli $(54,55)$. This homeostatic IL-22 induction, even in the absence of overt disease, helps to limit inflammatory colitis and has been correlated with mucosal healing in inflammatory bowel disease (IBD) $(83,84)$. Production of IBD-associated TNF-like ligand 1A (TL1A) by intestinal mononuclear phagocytes is a central regulator of ILC3 production of IL-22 and mediates protection during acute colitis (85). Similarly, ILC3-dependent protection occurs at other mucosal sites, including the lung, where commensal-dependent ILC3s protect against Streptococcus pneumoniae (86) and promote oropharyngeal barrier defense through IL-17 and IL-22 (87).

In addition to these direct effects, ILC3 production of IL-22 can also indirectly limit bacterial dysbiosis and inflammation at the gut barrier. The IBD-linked gene FUT2 is strongly induced in the intestinal epithelium by IL-22. The fucosyltransferase encoded by FUT2 is responsible for fucosylation of oligosaccharides on epithelial cells and provides a key nutrient for establishing a healthy and diverse microbiome (88-90). Mice lacking fucosyltransferase 2 have abnormal barrier function that renders the host more susceptible to enteric bacterial infections. Thus, by providing a nutrient substrate, ILC3-derived IL-22 promotes a healthy microbiota and colonization resistance to inflammation by potential pathobionts.

ILC2s play a critical role in coordinating the inflammatory response to helminth infection in the lung and gut. ILC2s secrete the type 2 cytokines IL- 5 and IL-13, which promote mucus and antimicrobial peptide $(\operatorname{RELM} \beta)$ production by intestinal goblet cells, limiting parasitic infections $(15,17,91$, 92). Recently, tuft cells were identified as the primary source of intestinal IL-25, which promotes ILC2 production of IL-13 (93-95). Tuft cells expand at weaning, when the increased abundance of polysaccharides in the intestine renders the host susceptible to gut infections $(96,97)$. In the case of Tritrichomonas infection, microbe-derived succinate actively triggers chemosensory receptors on tuft cells to induce IL-25 production. This microbe/IL-25/ILC2 circuit also enables ILC2 production of IL-13, which then drives stem cell proliferation, 


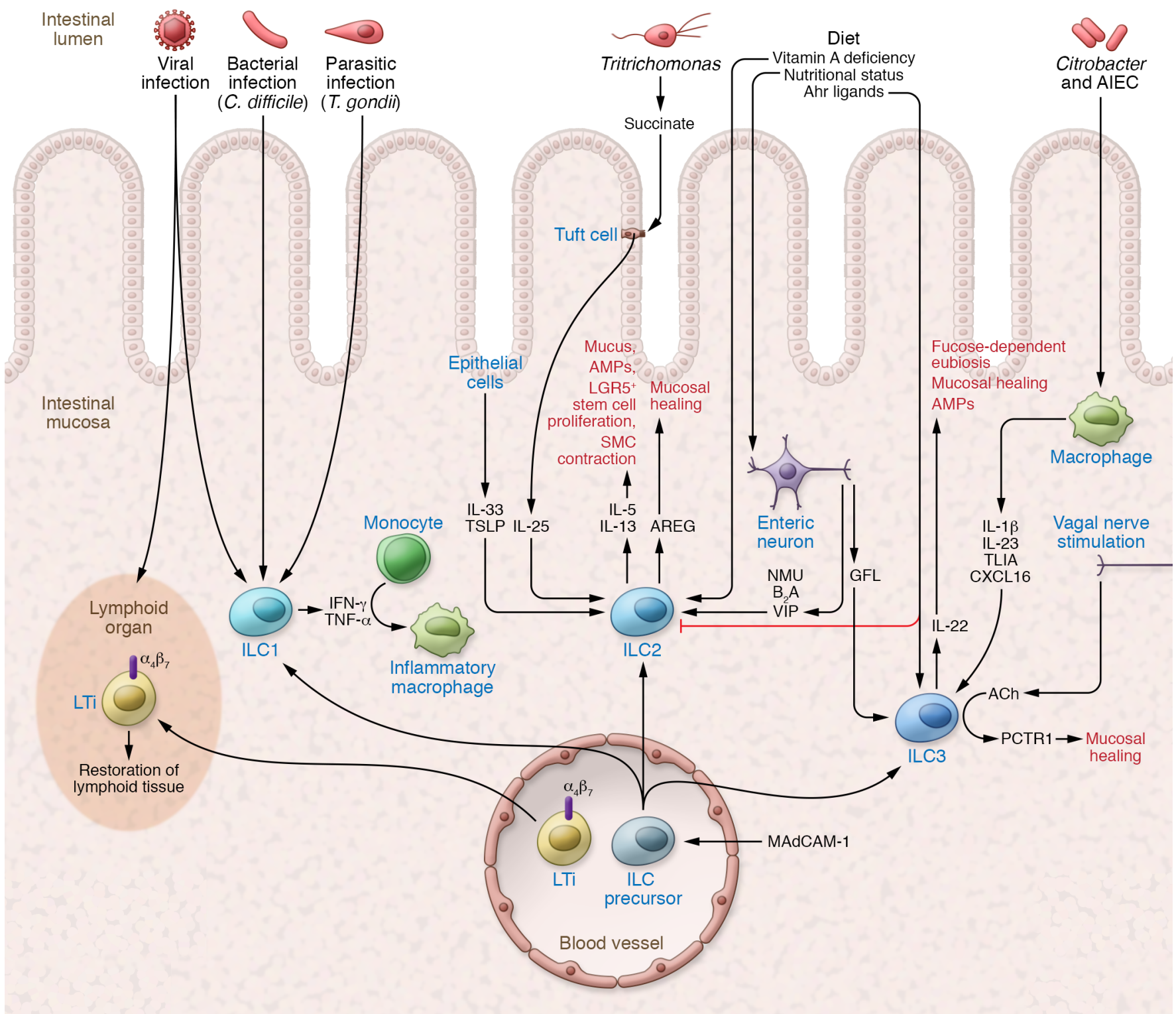

Figure 1. Tissue ILCs limit inflammation and promote restoration. ILCs serve as central mediators limiting inflammation from infectious and environmental triggers. Dietary as well as neuronal factors coordinate the response of ILC2s and ILC3s in the lamina propria. LTi cells play a critical role in restoring lymphoid tissue following viral infection. AREG, IL-22, and PCTR1 promote tissue repair and limit the impact of inflammation in the tissue. AIEC, adherent-invasive E. coli; GFL, GDNF family ligands; SMC, smooth muscle cell.

villous lengthening, and smooth muscle contraction to physically remove helminth infection. This "weep and sweep" response and remodeling of the intestine enforces compartmentalization and limits inflammation.

Finally, mucosal ILC1s also play a role in barrier defense, mounting a prototypical Th1-like response against pathogenic infections. During Toxoplasma gondii infection, ILC1s are the main producers of IFN- $\gamma$ and TNF- $\alpha$ (11), and during intestinal Clostridium difficile infections, ILC1s mediate protection via T-BET and IFN- $\gamma$ production (98). Tissue-resident ILC1s similarly serve an essential role in viral infection, enabling rapid production of IFN- $\gamma$ to limit early viral burden (99). Furthermore, the transfer of ILC precursors into alymphoid mice promotes the recruitment of monocytes and helps limit extensive inflammation (11). Collec- tively, these data reveal an essential role for ILCs in limiting tissue inflammation and promoting barrier defense.

\section{ILCs promote lymphoid and mucosal tissue restoration}

Tissue ILCs are also positioned to promote tissue and organ repair of both lymphoid and nonlymphoid structures (Figure 2). In addition to their role in fetal lymphoid tissue organogenesis, ILC3s are critical for restoring and maintaining tissue integrity following injury. After a viral infection, for example, LTi cells provide $\mathrm{LT} \alpha_{1} \beta_{2}$-dependent signaling in stromal cells to restore lymphoid tissue architecture (23). ILC-dependent restoration of immune tissue is also seen following radiation-induced thymic damage, wherein ILC3s promote thymic epithelial restoration via IL-22 


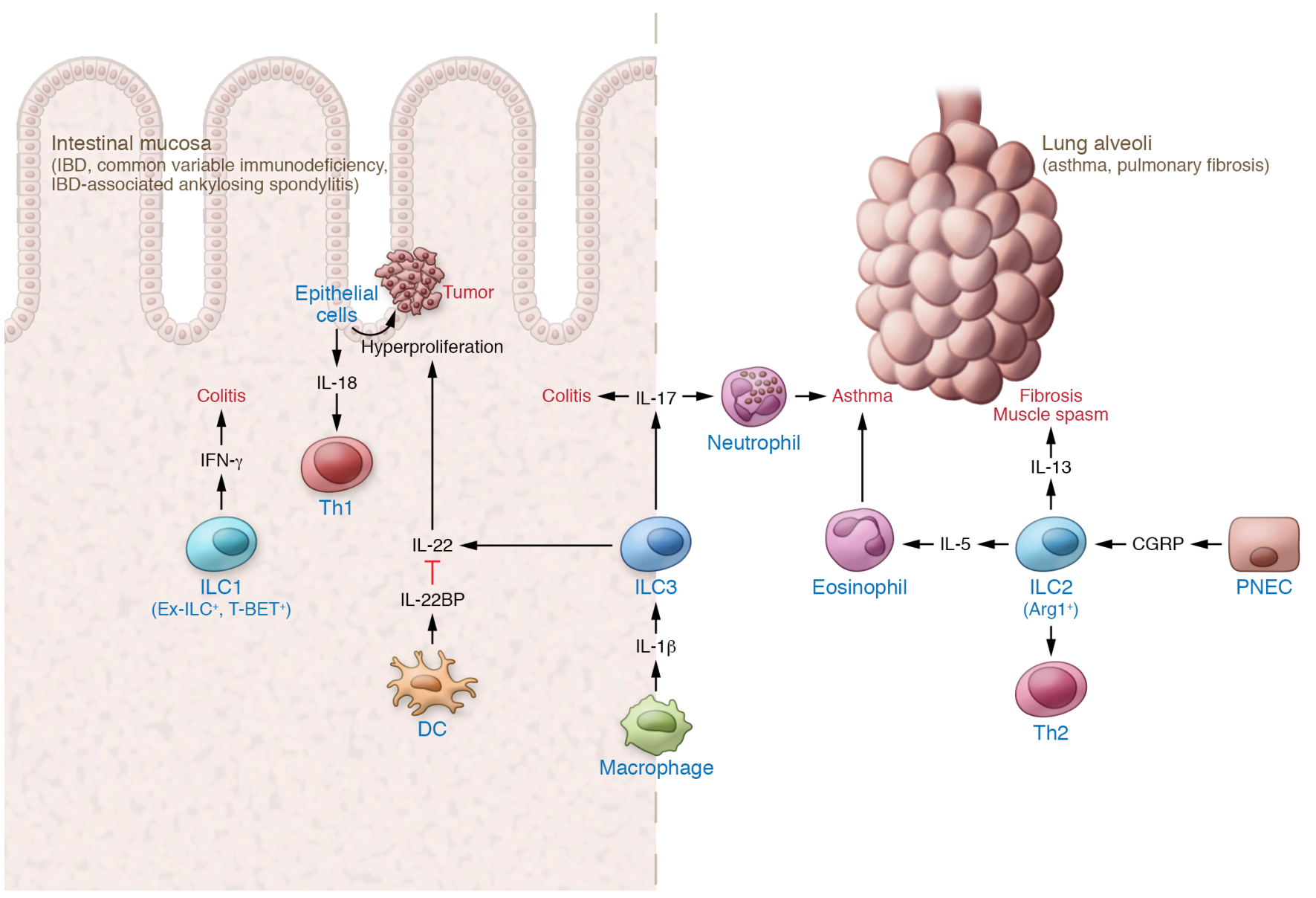

Figure 2. Tissue ILCs in chronic inflammation. Sustained activation of ILC effector functions promote inflammation. Restorative functions of IL-22 can lead to aberrant epithelial proliferation and IL-18 production to sustain an inflammatory Th1 response. ILC2 responses recruit eosinophil effectors, trigger smooth muscle contraction, and promote fibrosis.

(100). In addition, RANK-dependent signals from ILC3 support the maturation of $\mathrm{AIRE}^{+}$medullary thymic epithelial cells (101). This support of lymphoid tissue repair underlies an essential role for ILC3 in restoring tissue integrity and could serve as an important therapeutic target following total-body irradiation.

Ablation therapy prior to stem cell transplantation has also helped to reveal a critical role for ILCs in nonlymphoid tissue repair and preservation of mucosal barrier function. In particular, radioresistant ILC3s promote intestinal barrier function by supporting epithelial stem cells via production of IL-22 (102). IL-22 signaling at the intestinal crypt base triggers a STAT3-dependent antiapoptotic proliferative response in $\mathrm{Lgr5}^{+}$stem cells, promoting barrier repair and function (103). Moreover, in addition to the proliferative response, IL-22 induces STAT3 transcription of genes involved in radiation-induced damage response (104). This tissue-restorative function of ILC3s is not limited to radiation-induced damage, as ILC3s also upregulate IL-22 production following chemical and infectious damage to the lung and gut $(84,105,106)$. A similar effect is also seen in human IBD, where increased ILC3-derived IL-22 correlates with increased mucosal healing (84).

ILC2s also contribute to the tissue reparative response. ILC2 production of IL-13 promotes the accumulation of alternatively activated macrophages in both adipose and infected tissue wherein they promote metabolic control and wound healing (107, 108). This process can mediate hepatic stellate cell activation in liver tissue remodeling and fibrosis $(109,110)$. Type 2 cytokines can also mediate tissue repair by acting directly on nonmyeloid parenchymal cells to facilitate muscle regeneration (111). Moreover, IL-13 production by ILC2s acts directly upon IL-13Ra1 on intestinal stem cells to promote $\beta$-catenin pathway-dependent renewal (112). In addition to classical type 2 cytokine production, ILC2s produce the EGF family member amphiregulin (AREG) in response to IL-33 to support tissue regeneration (56). Although ILC2s do not directly block influenza viral infection in the lung, IL-33 induction of AREG (but not IL-13) plays a crucial role in survival by restoring airway integrity (113). An analogous IL-33/ILC2/AREG pathway also plays a critical role in intestinal repair, linking mucosal ILCs with a central EGF pathway critical in supporting intestinal epithelial cell renewal (114). Autocrine production of IL-9 amplifies IL-5, IL-13, and AREG production following helminth infection in the lung and promotes tissue repair in the recovery phase of the infec-

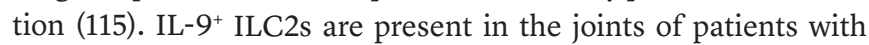
quiescent rheumatoid arthritis, and treatment with IL-9 promotes ILC2-dependent Treg activation and resolution of inflammation in mouse models (116), but further studies are needed to explore the potential for ILC2-based therapeutic strategies. 


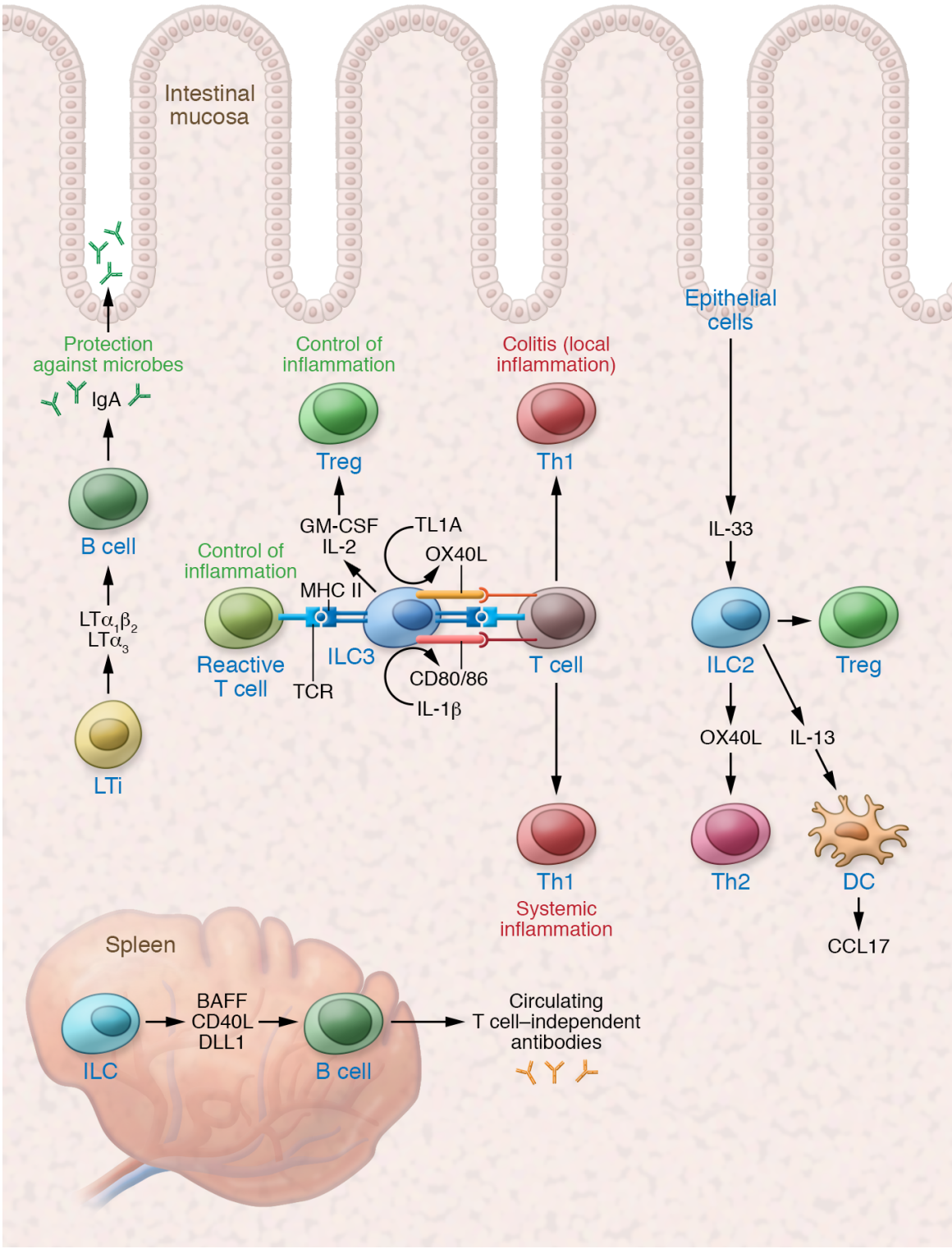

Figure 3. ILCs balance inflammatory and reparative adaptive immunity. LTi cells play a critical role in maintaining mucosal and systemic antibody production. MHC II+ ILC3s can limit the response of commensal-reactive T cells at steady state, but increases in TL1A or IL-1 $\beta$ can promote the activation of local and systemic inflammatory T cells, respectively. IL-33 induction of OX4OL by ILC2s can support tissue Th2 and Treg responses.

\section{ILCs in chronic inflammation: a double-edged sword}

The same characteristics and effector functions of tissue ILCs that limit inflammation and promote tissue repair can have pathogenic effects during chronic activation. Sustained production of IL-22 following $T$. gondii infection can trigger epithelial production of IL-18 and sustain a Th1 inflammatory loop (117), enabling collateral cross-reactive adaptive immunity against commensal microbial antigens and sustaining intestinal inflammation (118). Furthermore, chronic overproduction of IL-22 in RAG-deficient mice infected with Helicobacter hepaticus sustains epithelial hyperproliferation and, in the presence of the carcinogen azoxymethane, drives the development of colorectal cancer (119). The IL-22- binding protein (IL-22BP) produced by dendritic cells provides a tissue-restricted brake for this hyperproliferative response and limits the potential for tumorigenesis in both colitis-associated cancer and mice carrying a dominant mutation in the adenomatous polyposis coli (Apc) gene (120). In the absence of IL-22BP, mice susceptible to epithelial proliferation have increased tumor formation. Thus, even tissue-restorative responses of ILCs need to be tightly regulated to prevent damage associated with chronic activation.

A similar dichotomy is noted for ILC2s, which are recognized as the primary producers of type 2 effector responses in asthma and pulmonary fibrosis (121). Through the production of IL-5, ILC2s play a central role in recruiting eosinophils, the main effectors of allergic inflammatory disease, to the lung. Furthermore, smooth muscle contraction and mucus production induced by IL-13 can trigger allergic asthma symptoms, including respiratory spasms and mucus plugging. Sustained production of IL-13 also contributes to increased collagen deposition, resulting in chronic fibrotic tissue damage $(122,123)$. Constitutive expression of TL1A mimicking chronic inflammation triggers ILC2- and IL-13-dependent small intestinal and lung tissue allergic pathology (124). Supporting the potential role for ILC2 in chronic pathology, dermal and circulating ILC2s are elevated in patients with systemic sclerosis, and cell counts correlate with dermal and pulmonary fibrosis (125). Profibrotic functions of ILC2 occur in multiple organs, including the skin (126) and the kidney (127), and increased circulating ILC2s have been reported in patients with rheumatoid arthritis (128). Thus, chronic activation of ILC2s can lead to a sustained cycle of type 2 allergic responses and fibrotic disease pathology.

Local microenvironment cues can reprogram ILCs in situ to promote inflammation. NLRP3-dependent IL-1 $\beta$ production by macrophages during lung inflammation expands tissue ILC3s and increases IL-17 production, resulting in airway hyperresponsiveness (129). Similarly, in the intestine, chronic infection induces resident mononuclear phagocyte (MNP) production of IL-23, which can drive accumulation of proinflammatory ROR $\gamma \mathrm{t}$-dependent ILCs that express both IL-17A and IFN- $\gamma$ $(59,130)$. Transcriptional plasticity may underlie this transition in effector function. The induction of T-BET expression in ILC3s can antagonize ROR $\gamma \mathrm{t}$ expression and induce proinflammatory ex-ILC3 ILC1s that produce IFN- $\gamma(30,33)$. In human IBD, while an initial increase in ILC3 production of IL-22 correlates with mucosal healing (84), chronic colitis may reflect a transition from 
tissue-repairing ILC3s to inflammatory ex-ROR $\gamma \mathrm{t}^{+}$ILC1s $(10,131)$. The intestinal microenvironment and microbiota may enable the gut to serve as a portal for this transition. Intestinal biopsies from patients with IBD-associated ankylosing spondylitis showed an expansion of T-BET ${ }^{+}$ILC3s (132), and the expansion of TL1A-producing CX3CR1 ${ }^{+}$MNPs promoted T-BET ${ }^{+}$ILC3 activation in the synovial tissue (133). A parallel population of ex-ILC3s are also enriched in the synovial fluid of patients with psoriatic arthritis. Additional studies are needed in humans to define potential targetable tissue-specific cues that promote inflammatory ILCs in situ.

\section{ILC regulation of adaptive immunity and T cell inflammation}

ILCs have evolved to coordinate tissue-specific adaptive immunity (Figure 3). At barrier surfaces, they promote compartmentalization and limit tissue inflammation. For example, LTi cells provide survival and stimulation factors for B cells $(25,134)$. In the intestine, ILC3 expression of several TNF superfamily members (including $\mathrm{LT} \alpha_{1} \beta_{2}, \mathrm{LT} \alpha_{3}$, BAFF, APRIL, and LIGHT) plays a critical role in supporting homeostatic production of intestinal IgA. ILC3-derived $\mathrm{LT} \alpha_{1} \beta_{2}$ is required for T cell-independent IgA, while ILC3-derived $\mathrm{LT} \alpha_{3}$ can compensate for the absence of T cells (25). Systemically, splenic ILCs provide BAFF, CD4OL, and DLL1 to support innatelike B cell antibody production against circulating antigens (135). ILC support of humoral immunity, therefore, functions to promote compartmentalization and limit intestinal inflammation.

Tissue ILC3s can also regulate local T cell effectors. LTi expression of OX4OL and CD3OL supports homeostatic persistence of tissue $\mathrm{CD}^{+}$memory $\mathrm{T}$ cells (136). In addition, induction of ILC3-derived IL-22 by adherent microbiota triggers epithelial cell regulation of IL-17 production by intestinal $\mathrm{T}$ cells (54). Under homeostatic conditions, commensal microbiota and IL-1 $\beta$ also stimulate ILC3 production of GM-CSF as well as IL-2, which act via nonredundant pathways to promote intestinal Tregs $(137,138)$. Antigen processing and presentation by $\mathrm{MHC} \mathrm{II}^{+} \mathrm{ILC} 3$ s provide a mechanism for antigen-specific regulation of commensal-specific $\mathrm{T}$ cells (139). While IL-1 $\beta$ can trigger splenic ILC3 expression of CD80 and CD86 to support T cell activation (140), the absence of these costimulatory molecules on intestinal ILC3s limits homeostatic T cell activation (141). Under an inflammatory microenvironment such as IBD, these regulatory mechanisms are reduced (138, 141). Moreover, increased TL1A, which is highly expressed by MNPs in IBD, can induce robust expression of the costimulatory molecule OX4OL on MHC II ${ }^{+}$ILC3s and drive colonic T cell-dependent inflammation (85). Additional studies are needed to elucidate the contribution of ILC3s to mucosal T cell immunity and their potential role as therapeutic targets.

ILC2s similarly play a critical role in mounting a robust Th2 response $(142,143)$. ILC2-derived IL-13 promotes migration of tissue dendritic cells to the draining lymph node to prime Th2 immunity and elicits tissue dendritic cell production of Th2 cellattracting chemokine CCL17 to potentiate memory Th2 responses (144). Evolving work has illustrated a potential role for tissue-derived cytokines serving as a checkpoint for type 2 immunity (145). With respect to ILC2 regulation of Th2 immunity, tissue-specific release of IL-33 during intestinal or lung inflammation can also upregulate OX4OL on ILC2s, which subsequently regulates pro- tective Th2 immunity and Treg expansion in situ (146). Collectively, these pathways enable tissue ILC-mediated control of adaptive immunity in balancing inflammatory and restorative tissue responses.

\section{Future directions: targeting ILCs in the treatment of human disease}

It is important to consider the limitation that the majority of studies examining ILC function in vivo were performed in the absence of adaptive immunity, which may impede their translatability to human pathophysiology and the generation of novel therapeutics. Potential redundancy with effector $\mathrm{T}$ and $\mathrm{B}$ cells may exist and may reflect quantitative or kinetic differences in preventing infection and limiting inflammation. For example, selective targeting of $\mathrm{NCR}^{+}$ILC3s during Citrobacter infection showed that this subset is dispensable in the presence of T cells $(147,148)$. Human data, however, are limited in addressing the selective function of ILCs. Surprisingly, T cell reconstitutions in patients with severe combined immunodeficiency following hematopoietic stem cell transplantation (HSCT) revealed no clear increase in clinical disease susceptibility despite persistently fewer ILCs (149). Despite these findings, the increased susceptibility to mucosal pathogens Candida and Mycobacterium in patients with biallelic disruption of the RORC gene may illustrate subtle, yet crucial, features of mucosal immunity for which ILCs are required (150). Additional studies are required to fully capture the critical tissue ILC role in preventing infection and limiting inflammation in human diseases.

Despite these limitations, there is convincing evidence for ILC function in tissue restoration. ILC3 production of IL-22 following HSCT is a critical factor in tissue healing. Illustrating a potential role for ILC3 as a biomarker of tissue healing following HSCT, clinical data revealed an inverse correlation between mucosal (102) and circulating ILC3s (151) with the severity of graftversus-host disease. Similarly, in humans with acute IBD, increased ILC3-derived IL-22 correlates with mucosal healing - a critical clinical endpoint in IBD (84). In addition, AREG production following an acute asthma attack promotes tissue remodeling (152). As reviewed above, microbiota- and microbial-derived metabolic factors serve as potential therapeutic regulators of these tissue-restorative functions of ILCs. Additional studies in humans are needed to understand both diagnostic and therapeutic strategies to promote ILC3-based healing.

An important consideration with these approaches, however, is to limit inflammation associated with sustained ILC response. With the commercial availability of specific biologic therapies targeting effector cytokines as well as key trafficking molecules, diagnostic immunophenotyping may enable more precise utilization of therapies. Recent studies showing both mucosal and systemic Th17/ILC3 activation in IBD-associated spondyloarthritis $(55,153)$ highlight the need to investigate the efficacy of anti-IL-12/23 therapy in this clinical phenotype. Moreover, since the gut may serve as the portal for inflammatory TL1A-producing macrophages and $\alpha_{4} \beta_{7}^{+}, \mathrm{T}^{-B E T}{ }^{+}$ILCs in the synovial fluid (132), additional studies are needed to know whether anti-TL1A therapy or blockade of gut-derived $\alpha_{4} \beta_{7}{ }^{+}$lymphocytes will limit extraintestinal ILC inflammation $(154,155)$. In addition, sustained inflammation and pulmonary production of IL-25 in idiopathic pulmonary fibrosis 
lead to the accumulation of ILC2s and IL-13-mediated fibrotic disease (122). Clinical evidence targeting IL-13 in adults supports this as a target in limiting inflammation and fibrosis associated with moderate to severe asthma $(156,157)$. Recent data showing a role for the microRNA miR-19 in regulating ILC2 gene expression networks highlight the potential for microRNA as a novel therapeutic target (158). Further studies are needed in humans to define diagnostic clinical and molecular signatures of ILC-mediated disease in guiding precision therapy.

One key to improving therapeutic approaches is a better understanding of ILC subsets in human disease. The expansion of inflammatory ILCs in chronic IBD $(10,131)$ may reflect plasticity between ILC3s and ILC1s. T-BET ${ }^{+}$inflammatory ILCs are also expanded in patients with common variable immunodeficiency (159). The expansion of NKp $44^{+}$ILC3s in the dermal tissue from psoriatic lesions may similarly reflect this plasticity, and their reduction following successful treatment with TNF- $\alpha$ blockade therapy reveals the potential therapeutic relevance of understanding the interplay between ILC3 and ILC1 pathways (160). Pre- liminary data show that the expansion of inflammatory ILC3s in multiple sclerosis is reduced following anti-CD25 therapy (161), but additional analysis of these populations from inflammatory tissue is needed to define novel molecular targets (162). Clinical studies coupled with emerging sequencing technologies that offer comprehensive genomic characterization of tissue-specific ILC subsets will be critical in identifying ILC targets that can be therapeutically manipulated to treat human disease.

\section{Acknowledgments}

This work was supported in part by NIH Medical Scientist Training Grant T32GM07739 ( to JGC), NIH 1R03DK111852 (to RSL), NIH 1R01 DK114252 (to RSL), the New York Crohn's Foundation (to RSL), the Charina Foundation (to RSL), and the Kenneth Rainin Foundation (to RSL).

Address correspondence to: Randy S. Longman, Belfer Research Building, Room 714, 413 East 69th Street, New York, New York, USA. Phone: 212.746.5077; Email: ral2006@med.cornell.edu.
1. Hernández PP, et al. Single-cell transcriptional analysis reveals ILC-like cells in zebrafish. $\mathrm{Sci}$ Immunol. 2018;3(29):eaau5265.

2. Vivier E, van de Pavert SA, Cooper MD, Belz GT. The evolution of innate lymphoid cells. Nat Immunol. 2016;17(7):790-794.

3. Cherrier M, Sawa S, Eberl G. Notch, Id2, and ROR $\gamma \mathrm{t}$ sequentially orchestrate the fetal development of lymphoid tissue inducer cells. J Exp Med. 2012;209(4):729-740.

4. Satoh-Takayama N, et al. IL-7 and IL-15 independently program the differentiation of intestinal CD3-NKp $46^{+}$cell subsets from Id2-dependent precursors. J Exp Med. 2010;207(2):273-280.

5. Hoyler T, et al. The transcription factor GATA-3 controls cell fate and maintenance of type 2 innate lymphoid cells. Immunity. 2012;37(4):634-648.

6. Serafini N, et al. Gata3 drives development of ROR $\gamma \mathrm{t}^{+}$group 3 innate lymphoid cells. J Exp Med. 2014;211(2):199-208.

7. Vivier E, et al. Innate lymphoid cells: 10 years on. Cell. 2018;174(5):1054-1066.

8. Cherrier DE, Serafini N, Di Santo JP. Innate lymphoid cell development: a T cell perspective. Immunity. 2018;48(6):1091-1103.

9. Daussy C, et al. T-bet and Eomes instruct the development of two distinct natural killer cell lineages in the liver and in the bone marrow. J Exp Med. 2014;211(3):563-577.

10. Bernink JH, et al. Human type 1 innate lymphoid cells accumulate in inflamed mucosal tissues. Nat Immunol. 2013;14(3):221-229.

11. Klose CSN, et al. Differentiation of type 1 ILCs from a common progenitor to all helperlike innate lymphoid cell lineages. Cell. 2014;157(2):340-356.

12. Fuchs A, et al. Intraepithelial type 1 innate lymphoid cells are a unique subset of IL-12and IL-15-responsive IFN- $\gamma$-producing cells. Immunity. 2013;38(4):769-781.

13. Gao Y, et al. Tumor immunoevasion by the con- version of effector NK cells into type 1 innate lymphoid cells. Nat Immunol. 2017;18(9):1004-1015.

14. Cortez VS, Fuchs A, Cella M, Gilfillan S, Colonna M. Cutting edge: Salivary gland NK cells develop independently of Nfil3 in steady-state. J Immunol. 2014;192(10):4487-4491.

15. Price AE, et al. Systemically dispersed innate IL-13-expressing cells in type 2 immunity. Proc Natl Acad Sci U S A . 2010;107(25):11489-11494.

16. Nussbaum JC, et al. Type 2 innate lymphoid cells control eosinophil homeostasis. Nature. 2013;502(7470):245-248.

17. Moro K, et al. Innate production of $\mathrm{T}(\mathrm{H}) 2$ cytokines by adipose tissue-associated c-Kit(+)Sca-1(+) lymphoid cells. Nature. 2010;463(7280):540-544.

18. Mjösberg J, et al. The transcription factor GATA3 is essential for the function of human type 2 innate lymphoid cells. Immunity. 2012;37(4):649-659.

19. Hams E, Locksley RM, McKenzie AN, Fallon PG. Cutting edge: IL-25 elicits innate lymphoid type 2 and type II NKT cells that regulate obesity in mice. J Immunol. 2013;191(11):5349-5353.

20. Brestoff JR, et al. Group 2 innate lymphoid cells promote beiging of white adipose tissue and limit obesity. Nature. 2015;519(7542):242-246.

21. Eberl G, Marmon S, Sunshine MJ, Rennert PD, Choi Y, Littman DR. An essential function for the nuclear receptor $\mathrm{ROR} \gamma(\mathrm{t})$ in the generation of fetal lymphoid tissue inducer cells. Nat Immunol. 2004;5(1):64-73.

22. Mebius RE. Organogenesis of lymphoid tissues. Nat Rev Immunol. 2003;3(4):292-303.

23. Scandella E, et al. Restoration of lymphoid organ integrity through the interaction of lymphoid tissue-inducer cells with stroma of the T cell zone. Nat Immunol. 2008;9(6):667-675.

24. Magri G, Cerutti A. Role of group 3 innate lymphoid cells in antibody production. Curr Opin Immunol. 2015;33:36-42.

25. Kruglov AA, et al. Nonredundant function of soluble LT $\alpha 3$ produced by innate lymphoid cells in intestinal homeostasis. Science. 2013;342(6163):1243-1246.

26. Cella M, et al. A human natural killer cell subset provides an innate source of IL-22 for mucosal immunity. Nature. 2009;457(7230):722-725.

27. Luci C, et al. Influence of the transcription factor RORgammat on the development of $\mathrm{NKp} 46^{+}$ cell populations in gut and skin. Nat Immunol. 2009;10(1):75-82.

28. Satoh-Takayama N, et al. Microbial flora drives interleukin 22 production in intestinal $\mathrm{NKp} 46^{+}$ cells that provide innate mucosal immune defense. Immunity. 2008;29(6):958-970.

29. Sanos SL, et al. ROR $\gamma \mathrm{t}$ and commensal microflora are required for the differentiation of mucosal interleukin 22-producing NKp46 $6^{+}$cells. Nat Immunol. 2009;10(1):83-91.

30. Klose CS, et al. A T-bet gradient controls the fate and function of CCR6-ROR $\gamma \mathrm{t}^{+}$innate lymphoid cells. Nature. 2013;494(7436):261-265.

31. Rankin LC, et al. The transcription factor T-bet is essential for the development of NKp46 ${ }^{+}$innate lymphocytes via the Notch pathway. Nat Immunol. 2013;14(4):389-395.

32. Colonna M. Innate lymphoid cells: diversity, plasticity, and unique functions in immunity. Immunity. 2018;48(6):1104-1117.

33. Vonarbourg C, et al. Regulated expression of nuclear receptor ROR $\gamma \mathrm{t}$ confers distinct functional fates to NK cell receptor-expressing ROR $\gamma \mathrm{t}(+)$ innate lymphocytes. Immunity. 2010;33(5):736-751.

34. Silver JS, et al. Inflammatory triggers associated with exacerbations of COPD orchestrate plasticity of group 2 innate lymphoid cells in the lungs. Nat Immunol. 2016;17(6):626-635.

35. Lim AI, et al. IL-12 drives functional plasticity of human group 2 innate lymphoid cells. J Exp Med. 2016;213(4):569-583.

36. Wang S, et al. Regulatory innate lymphoid cells control innate intestinal inflammation. Cell. 2017;171(1):201-216.e18.

37. Seehus CR, et al. Alternative activation generates 
IL-10 producing type 2 innate lymphoid cells. Nat Commun. 2017;8(1):1900.

38. Montaldo E, et al. Human ROR $\gamma \mathrm{t}(+) \mathrm{CD} 34(+)$ cells are lineage-specified progenitors of group $3 \mathrm{ROR} \gamma \mathrm{t}(+)$ innate lymphoid cells. Immunity. 2014;41(6):988-1000.

39. Scoville SD, et al. A progenitor cell expressing transcription factor ROR $\gamma$ t generates all human innate lymphoid cell subsets. Immunity. 2016;44(5):1140-1150.

40. Cella M, Otero K, Colonna M. Expansion of human NK-22 cells with IL-7, IL-2, and IL-1及 reveals intrinsic functional plasticity. Proc Natl Acad Sci U S A. 2010;107(24):10961-10966.

41. Bando JK, Liang HE, Locksley RM. Identification and distribution of developing innate lymphoid cells in the fetal mouse intestine. Nat Immunol. 2015;16(2):153-160

42. Li N, et al. Mass cytometry reveals innate lymphoid cell differentiation pathways in the human fetal intestine. J Exp Med. 2018;215(5):1383-1396.

43. Gasteiger G, Fan X, Dikiy S, Lee SY, Rudensky AY. Tissue residency of innate lymphoid cells in lymphoid and nonlymphoid organs. Science. 2015;350(6263):981-985.

44. Moro K, et al. Interferon and IL-27 antagonize the function of group 2 innate lymphoid cells and type 2 innate immune responses. Nat Immunol. 2016;17(1):76-86.

45. Ricardo-Gonzalez RR, et al. Tissue signals imprint ILC2 identity with anticipatory function. Nat Immunol. 2018;19(10):1093-1099.

46. Nussbaum K, et al. Tissue microenvironment dictates the fate and tumor-suppressive function of type 3 ILCs. J Exp Med. 2017;214(8):2331-2347.

47. Germain RN, Huang Y. ILC2s - resident lymphocytes pre-adapted to a specific tissue or migratory effectors that adapt to where they move? Curr Opin Immunol. 2019;56:76-81.

48. Yang J, Hu S, Zhao L, Kaplan DH, Perdew GH, Xiong N. Selective programming of CCR10(+) innate lymphoid cells in skin-draining lymph nodes for cutaneous homeostatic regulation. Nat Immunol. 2016;17(1):48-56.

49. Kim MH, Taparowsky EJ, Kim CH. Retinoic acid differentially regulates the migration of innate lymphoid cell subsets to the gut. Immunity. 2015;43(1):107-119.

50. Satoh-Takayama N, et al. The chemokine receptor CXCR6 controls the functional topography of interleukin-22 producing intestinal innate lymphoid cells. Immunity. 2014;41(5):776-788.

51. Mackay LK, et al. Hobit and Blimp1 instruct a universal transcriptional program of tissue residency in lymphocytes. Science. 2016;352(6284):459-463.

52. Fung TC, et al. Lymphoid-tissue-resident commensal bacteria promote members of the IL-10 cytokine family to establish mutualism. Immunity. 2016;44(3):634-646.

53. Atarashi $\mathrm{K}$, et al. Th17 cell induction by adhesion of microbes to intestinal epithelial cells. Cell. 2015;163(2):367-380.

54. Sano T, et al. An IL-23R/IL-22 circuit regulates epithelial serum amyloid a to promote local effector Th17 responses. Cell. 2016;164(1-2):324.

55. Viladomiu M, et al. IgA-coated E. coli enriched in Crohn's disease spondyloarthritis promote TH17-dependent inflammation. Sci Transl Med. 2017;9(376):eaaf9655.

56. Monticelli LA, et al. Innate lymphoid cells promote lung-tissue homeostasis after infection with influenza virus. Nat Immunol. 2011;12(11):1045-1054.

57. Kernbauer E, Ding Y, Cadwell K. An enteric virus can replace the beneficial function of commensal bacteria. Nature. 2014;516(7529):94-98.

58. Gury-BenAri M, et al. The spectrum and regulatory landscape of intestinal innate lymphoid cells are shaped by the microbiome. Cell. 2016;166(5):1231-1246.e13.

59. Buonocore $S$, et al. Innate lymphoid cells drive interleukin-23-dependent innate intestinal pathology. Nature. 2010;464(7293):1371-1375.

60. Kiss EA, et al. Natural aryl hydrocarbon receptor ligands control organogenesis of intestinal lymphoid follicles. Science. 2011;334(6062):1561-1565.

61. Robinette ML, et al. Transcriptional programs define molecular characteristics of innate lymphoid cell classes and subsets. Nat Immunol. 2015;16(3):306-317

62. Qiu J, et al. The aryl hydrocarbon receptor regulates gut immunity through modulation of innate lymphoid cells. Immunity. 2012;36(1):92-104.

63. Lee JS, et al. AHR drives the development of gut ILC22 cells and postnatal lymphoid tissues via pathways dependent on and independent of Notch. Nat Immunol. 2011;13(2):144-151.

64. Qiu J, et al. Group 3 innate lymphoid cells inhibit T-cell-mediated intestinal inflammation through aryl hydrocarbon receptor signaling and regulation of microflora. Immunity. 2013;39(2):386-399.

65. Li S, et al. Aryl hydrocarbon receptor signaling cell intrinsically inhibits intestinal group 2 innate lymphoid cell function. Immunity. 2018;49(5):915-928.e5.

66. Zelante T, et al. Tryptophan catabolites from microbiota engage aryl hydrocarbon receptor and balance mucosal reactivity via interleukin-22. Immunity. 2013;39(2):372-385.

67. Gomez de Agüero M, et al. The maternal microbiota drives early postnatal innate immune development. Science. 2016;351(6279):1296-1302.

68. Mielke LA, et al. Retinoic acid expression associates with enhanced IL-22 production by $\gamma \delta$ T cells and innate lymphoid cells and attenuation of intestinal inflammation. J Exp Med. 2013;210(6):1117-1124.

69. van de Pavert SA, et al. Maternal retinoids control type 3 innate lymphoid cells and set the offspring immunity. Nature. 2014;508(7494):123-127.

70. Spencer SP, et al. Adaptation of innate lymphoid cells to a micronutrient deficiency promotes type 2 barrier immunity. Science. 2014;343(6169):432-437.

71. Wilhelm C, et al. Critical role of fatty acid metabolism in ILC2-mediated barrier protection during malnutrition and helminth infection. JExp Med. 2016;213(8):1409-1418

72. Monticelli LA, et al. Arginase 1 is an innate lymphoid-cell-intrinsic metabolic checkpoint controlling type 2 inflammation. Nat Immunol. 2016;17(6):656-665.

73. Veiga-Fernandes $\mathrm{H}$, et al. Tyrosine kinase receptor RET is a key regulator of Peyer's patch organ- ogenesis. Nature. 2007;446(7135):547-551.

74. Klose CSN, et al. The neuropeptide neuromedin U stimulates innate lymphoid cells and type 2 inflammation. Nature. 2017;549(7671):282-286.

75. Wallrapp A, et al. The neuropeptide NMU amplifies ILC2-driven allergic lung inflammation. Nature. 2017;549(7672):351-356.

76. Cardoso V, et al. Neuronal regulation of type 2 innate lymphoid cells via neuromedin U. Nature. 2017;549(7671):277-281.

77. Sui P, et al. Pulmonary neuroendocrine cells amplify allergic asthma responses. Science. 2018;360(6393):eaan8546.

78. Moriyama S, et al. $\beta_{2}$-adrenergic receptormediated negative regulation of group 2 innate lymphoid cell responses. Science. 2018;359(6379):1056-1061.

79. Ibiza S, et al. Glial-cell-derived neuroregulators control type 3 innate lymphoid cells and gut defence. Nature. 2016;535(7612):440-443

80. Dalli J, Colas RA, Arnardottir H, Serhan CN Vagal regulation of group 3 innate lymphoid cells and the immunoresolvent PCTR1 controls infection resolution. Immunity. 2017;46(1):92-105.

81. Zheng Y, et al. Interleukin-22 mediates early host defense against attaching and effacing bacterial pathogens. Nat Med. 2008;14(3):282-289.

82. Sonnenberg GF, Artis D. Innate lymphoid cells in the initiation, regulation and resolution of inflammation. Nat Med. 2015;21(7):698-708.

83. Guo X, Liang Y, Zhang Y, Lasorella A, Kee BL, Fu YX. Innate lymphoid cells control early colonization resistance against intestinal pathogens through ID2-dependent regulation of the microbiota. Immunity. 2015;42(4):731-743.

84. Longman RS, et al. CX(3)CR1(+) mononuclear phagocytes support colitis-associated innate lymphoid cell production of IL-22. J Exp Med. 2014;211(8):1571-1583.

85. Castellanos JG, et al. Microbiota-induced TNFlike ligand $1 \mathrm{~A}$ drives group 3 innate lymphoid cell-mediated barrier protection and intestinal $\mathrm{T}$ cell activation during colitis. Immunity. 2018;49(6):1077-1089.e5.

86. Gray J, Oehrle K, Worthen G, Alenghat T, Whitsett J, Deshmukh H. Intestinal commensal bacteria mediate lung mucosal immunity and promote resistance of newborn mice to infection. Sci Transl Med. 2017;9(376):eaaf9412.

87. Gladiator A, Wangler N, Trautwein-Weidner K, LeibundGut-Landmann S. Cutting edge: IL-17-secreting innate lymphoid cells are essential for host defense against fungal infection. J Immunol. 2013;190(2):521-525.

88. Pham TA, et al. Epithelial IL-22RA1-mediated fucosylation promotes intestinal colonization resistance to an opportunistic pathogen. Cell Host Microbe. 2014;16(4):504-516.

89. Goto Y, et al. Innate lymphoid cells regulate intestinal epithelial cell glycosylation. Science. 2014;345(6202):1254009.

90. Pickard JM, et al. Rapid fucosylation of intestinal epithelium sustains host-commensal symbiosis in sickness. Nature. 2014;514(7524):638-641.

91. Neill DR, et al. Nuocytes represent a new innate effector leukocyte that mediates type-2 immunity. Nature. 2010;464(7293):1367-1370.

92. Saenz SA, et al. IL25 elicits a multipotent progen- 
itor cell population that promotes $\mathrm{T}(\mathrm{H}) 2$ cytokine responses. Nature. 2010;464(7293):1362-1366.

93. Gerbe F, et al. Intestinal epithelial tuft cells initiate type 2 mucosal immunity to helminth parasites. Nature. 2016;529(7585):226-230.

94. von Moltke J, Ji M, Liang HE, Locksley RM. Tuft-cell-derived IL-25 regulates an intestinal ILC2-epithelial response circuit. Nature. 2016;529(7585):221-225.

95. Howitt MR, et al. Tuft cells, taste-chemosensory cells, orchestrate parasite type 2 immunity in the gut. Science. 2016;351(6279):1329-1333.

96. Nadjsombati MS, et al. Detection of succinate by intestinal tuft cells triggers a type 2 innate immune circuit. Immunity. 2018;49(1):33-41.e7.

97. Schneider C, et al. A metabolite-triggered tuft cell-ILC2 circuit drives small intestinal remodeling. Cell. 2018;174(2):271-284.e14.

98. Abt MC, et al. Innate immune defenses mediated by two ILC subsets are critical for protection against acute clostridium difficile infection. Cell Host Microbe. 2015;18(1):27-37.

99. Weizman OE, et al. ILC1 confer early host protection at initial sites of viral infection. Cell. 2017;171(4):795-808.e12.

100.Dudakov JA, et al. Interleukin-22 drives endogenous thymic regeneration in mice. Science. 2012;336(6077):91-95.

101.Rossi SW, et al. RANK signals from CD4(+)3(-) inducer cells regulate development of Aireexpressing epithelial cells in the thymic medulla. JExp Med. 2007;204(6):1267-1272.

102. Hanash AM, et al. Interleukin-22 protects intestinal stem cells from immune-mediated tissue damage and regulates sensitivity to graft versus host disease. Immunity. 2012;37(2):339-350.

103. Lindemans $C A$, et al. Interleukin-22 promotes intestinal-stem-cell-mediated epithelial regeneration. Nature. 2015;528(7583):560-564.

104.Gronke K, et al. Interleukin-22 protects intestinal stem cells against genotoxic stress. Nature. 2019;566(7743):249-253.

105. Sonnenberg GF, Nair MG, Kirn TJ, Zaph C, Fouser LA, Artis D. Pathological versus protective functions of IL-22 in airway inflammation are regulated by IL-17A. JExp Med. 2010;207(6):1293-1305.

106.Sonnenberg GF, Monticelli LA, Elloso MM, Fouser LA, Artis D. CD4(+) lymphoid tissue-inducer cells promote innate immunity in the gut. Immunity. 2011;34(1):122-134.

107. Molofsky AB, et al. Innate lymphoid type 2 cells sustain visceral adipose tissue eosinophils and alternatively activated macrophages. J Exp Med. 2013;210(3):535-549.

108. Chen F, et al. An essential role for TH2-type responses in limiting acute tissue damage during experimental helminth infection. Nat Med. 2012;18(2):260-266

109. McHedlidze T, et al. Interleukin-33-dependent innate lymphoid cells mediate hepatic fibrosis. Immunity. 2013;39(2):357-371.

110.Forkel M, et al. Composition and functionality of the intrahepatic innate lymphoid cell-compartment in human nonfibrotic and fibrotic livers. Eur J Immunol. 2017;47(8):1280-1294.

111. Heredia JE, et al. Type 2 innate signals stimulate fibro/adipogenic progenitors to facilitate muscle regeneration. Cell. 2013;153(2):376-388.

112. Zhu P, et al. IL-13 secreted by ILC2s promotes the self-renewal of intestinal stem cells through circular RNA circPan3. Nat Immunol. 2019;20(2):183-194.

113. Zaiss DMW, Gause WC, Osborne LC, Artis D. Emerging functions of amphiregulin in orchestrating immunity, inflammation, and tissue repair. Immunity. 2015;42(2):216-226.

114. Monticelli LA, Osborne LC, Noti M, Tran SV, Zaiss DM, Artis D. IL-33 promotes an innate immune pathway of intestinal tissue protection dependent on amphiregulin-EGFR interactions. Proc Natl Acad Sci U S A. 2015;112(34):10762-10767.

115. Turner JE, et al. IL-9-mediated survival of type 2 innate lymphoid cells promotes damage control in helminth-induced lung inflammation. J Exp Med. 2013;210(13):2951-2965.

116. Rauber S, et al. Resolution of inflammation by interleukin-9-producing type 2 innate lymphoid cells. Nat Med. 2017;23(8):938-944.

117. Muñoz M, et al. Interleukin-22 induces interleukin-18 expression from epithelial cells during intestinal infection. Immunity. 2015;42(2):321-331.

118. Hand TW, et al. Acute gastrointestinal infection induces long-lived microbiota-specific $\mathrm{T}$ cell responses. Science. 2012;337(6101):1553-1556.

119. Kirchberger S, et al. Innate lymphoid cells sustain colon cancer through production of interleukin-22 in a mouse model. JExp Med. 2013;210(5):917-931.

120. Huber S, et al. IL-22BP is regulated by the inflammasome and modulates tumorigenesis in the intestine. Nature. 2012;491(7423):259-263.

121. Monticelli LA, Sonnenberg GF, Artis D. Innate lymphoid cells: critical regulators of allergic inflammation and tissue repair in the lung. Curr Opin Immunol. 2012;24(3):284-289.

122. Hams E, et al. IL-25 and type 2 innate lymphoid cells induce pulmonary fibrosis. Proc Natl Acad Sci U S A. 2014;111(1):367-372.

123. Chang YJ, et al. Innate lymphoid cells mediate influenza-induced airway hyper-reactivity independently of adaptive immunity. Nat Immunol. 2011;12(7):631-638.

124. Meylan F, et al. The TNF-family cytokine TL1A promotes allergic immunopathology through group 2 innate lymphoid cells. Mucosal Immunol. 2014;7(4):958-968.

125. Wohlfahrt T, et al. Type 2 innate lymphoid cell counts are increased in patients with systemic sclerosis and correlate with the extent of fibrosis. Ann Rheum Dis. 2016;75(3):623-626.

126. Rak GD, et al. IL-33-dependent group 2 innate lymphoid cells promote cutaneous wound healing. J Invest Dermatol. 2016;136(2):487-496.

127. Riedel JH, et al. IL-33-mediated expansion of type 2 innate lymphoid cells protects from progressive glomerulosclerosis. J Am Soc Nephrol. 2017;28(7):2068-2080.

128. Omata Y, et al. Group 2 innate lymphoid cells attenuate inflammatory arthritis and protect from bone destruction in mice. Cell Rep. 2018;24(1):169-180.

129. Kim HY, et al. Interleukin-17-producing innate lymphoid cells and the NLRP3 inflammasome facilitate obesity-associated airway hyperreactiv- ity. Nat Med.2014;20(1):54-61.

130. Garrett WS, et al. Communicable ulcerative colitis induced by T-bet deficiency in the innate immune system. Cell. 2007;131(1):33-45.

131. Geremia A, et al. IL-23-responsive innate lymphoid cells are increased in inflammatory bowel disease. J Exp Med. 2011;208(6):1127-1133.

132. Ciccia F, et al. Type 3 innate lymphoid cells producing IL-17 and IL-22 are expanded in the gut, in the peripheral blood, synovial fluid and bone marrow of patients with ankylosing spondylitis. Ann Rheum Dis. 2015;74(9):1739-1747.

133. Ciccia F, et al. Proinflammatory CX3CR1 ${ }^{+}$ $\mathrm{CD} 9^{+}$tumor necrosis factor-like molecule $1 \mathrm{~A}^{+}$interleukin- $23^{+}$monocytes are expanded in patients with ankylosing spondylitis and modulate innate lymphoid cell 3 immune functions. Arthritis Rheumatol. 2018;70(12):2003-2013.

134. Tsuji M, et al. Requirement for lymphoid tissueinducer cells in isolated follicle formation and $\mathrm{T}$ cell-independent immunoglobulin A generation in the gut. Immunity. 2008;29(2):261-271.

135. Magri G, et al. Innate lymphoid cells integrate stromal and immunological signals to enhance antibody production by splenic marginal zone $B$ cells. Nat Immunol. 2014;15(4):354-364.

136. Withers DR, et al. Cutting edge: lymphoid tissue inducer cells maintain memory CD4 T cells within secondary lymphoid tissue. JImmunol. 2012;189(5):2094-2098.

137. Mortha A, et al. Microbiota-dependent crosstalk between macrophages and ILC3 promotes intestinal homeostasis. Science. 2014;343(6178):1249288

138. Zhou L, et al. Innate lymphoid cells support regulatory $\mathrm{T}$ cells in the intestine through interleukin-2. Nature. 2019;568(7752):405-409.

139. Hepworth MR, et al. Innate lymphoid cells regulate $\mathrm{CD} 4^{+} \mathrm{T}$-cell responses to intestinal commensal bacteria. Nature. 2013;498(7452):113-117.

140.von Burg N, et al. Activated group 3 innate lymphoid cells promote T-cell-mediated immune responses. Proc Natl Acad Sci U S A. 2014;111(35):12835-12840.

141. Hepworth MR, et al. Immune tolerance. Group 3 innate lymphoid cells mediate intestinal selection of commensal bacteria-specific CD4(+) T cells. Science. 2015;348(6238):1031-1035.

142. Halim TY, et al. Group 2 innate lymphoid cells are critical for the initiation of adaptive $\mathrm{T}$ helper 2 cell-mediated allergic lung inflammation. Immunity. 2014;40(3):425-435.

143. Oliphant CJ, et al. MHCII-mediated dialog between group 2 innate lymphoid cells and CD4(+) T cells potentiates type 2 immunity and promotes parasitic helminth expulsion. Immunity. 2014;41(2):283-295.

144. Halim TY, et al. Group 2 innate lymphoid cells license dendritic cells to potentiate memory TH2 cell responses. Nat Immunol. 2016;17(1):57-64.

145. Van Dyken SJ, et al. A tissue checkpoint regulates type 2 immunity. Nat Immunol. 2016;17(12):1381-1387.

146. Halim TYF, et al. Tissue-restricted adaptive type 2 immunity is orchestrated by expression of the costimulatory molecule OX4OL on group 2 innate lymphoid cells. Immunity. 2018;48(6):1195-1207.e6. 
147. Rankin LC, et al. Complementarity and redundancy of IL-22-producing innate lymphoid cells. Nat Immunol. 2016;17(2):179-186.

148.Song C, et al. Unique and redundant functions of NKp46 ${ }^{+}$ILC3s in models of intestinal inflammation. JExp Med. 2015;212(11):1869-1882.

149. Vely F, et al. Evidence of innate lymphoid cell redundancy in humans. Nat Immunol. 2016;17(11):1291-1299.

150. Okada S, et al. Impairment of immunity to Candida and Mycobacterium in humans with bi-allelic RORC mutations. Science. 2015;349(6248):606-613.

151. Munneke JM, et al. Activated innate lymphoid cells are associated with a reduced susceptibility to graft-versus-host disease. Blood. 2014;124(5):812-821.

152. Enomoto Y, et al. Tissue remodeling induced by hypersecreted epidermal growth factor and amphiregulin in the airway after an acute asthma attack.
JAllergy Clin Immunol. 2009;124(5):913-920.e1.

153. Ciccia F, et al. Interleukin-22 and interleukin-22-producing $\mathrm{NKp} 44^{+}$natural killer cells in subclinical gut inflammation in ankylosing spondylitis. Arthritis Rheum. 2012;64(6):1869-1878.

154. Ciccia F, Rizzo A, Guggino G, Bignone R, Galia M, Triolo G. Clinical efficacy of $\alpha 4$ integrin block with natalizumab in ankylosing spondylitis. Ann Rheum Dis. 2016;75(11):2053-2054.

155. Orlando A, et al. Clinical benefit of vedolizumab on articular manifestations in patients with active spondyloarthritis associated with inflammatory bowel disease. Ann Rheum Dis. 2017;76(9):e31.

156. Piper E, et al. A phase II placebo-controlled study of tralokinumab in moderate-to-severe asthma. Eur Respir J. 2013;41(2):330-338.

157. Corren J, et al. Lebrikizumab treatment in adults with asthma. $N$ Engl J Med. 2011;365(12):1088-1098.
158. Singh PB, et al. MicroRNA regulation of type 2 innate lymphoid cell homeostasis and function in allergic inflammation. J Exp Med. 2017;214(12):3627-3643

159. Cols M, et al. Expansion of inflammatory innate lymphoid cells in patients with common variable immune deficiency. J Allergy Clin Immunol. 2016;137(4):1206-1215.e6.

160.Villanova F, et al. Characterization of innate lymphoid cells in human skin and blood demonstrates increase of NKp $44^{+}$ILC3 in psoriasis. Invest Dermatol. 2014;134(4):984-991.

161. Perry JS, et al. Inhibition of LTi cell development by CD 25 blockade is associated with decreased intrathecal inflammation in multiple sclerosis. Sci Transl Med. 2012;4(145):145ra106.

162. Yudanin NA, et al. Spatial and temporal map ping of human innate lymphoid cells reveals elements of tissue specificity. Immunity. 2019;50(2):505-519.e4. 